\title{
Gated cardiac blood pool studies in atrial fibrillation: role of cycle length windowing
}

\author{
Jerold W. Wallis, Jack E. Juni ${ }^{*}$ and Lucy Wu \\ Division of Nuclear Medicine, University of Michigan Hospitals, Ann Arbor, Michigan, USA \\ Received May 16, 1990 and in revised form July 27, 1990
}

\begin{abstract}
Cycle length windowing is gaining increasing acceptance in gated blood pool imaging of patients with atrial fibrillation (AF). The goals of this study were: to assess differences of ejection fraction (EF) in AF with and without windowing and to determine how EF varied with cycle length in patients with AF. Twenty patients with AF were prospectively studied by gated blood pool imaging, with simultaneous collection in each patient of 5-7 studies with cycle length windows spanning the cycle length histogram. Each window accepted beats of only a narrow range of cycle lengths. EF was determined for each of the narrow cycle length windows as well as for the entire gated blood pool study without cycle length windowing. For every patient an average of the windowed EFs was compared with the non-windowed EF. EF values were similar (mean windowed: 46.6 ; nonwindowed: $45.5 ; P=0.16$ ), and there was a good correlation between the two techniques $(r=0.97)$. The data were then examined for a relationship of EF with cycle length. The difference from average windowed $\mathrm{EF}(\Delta \mathrm{EF})$ was calculated for each window and plotted vs. the cycle length of the center of each window. No predictable linear or nonlinear relationship of $\triangle \mathrm{EF}$ with window position was observed. Lack of predictable variation of EF with cycle length is likely due to lack of a predictable amount of ventricular filling for a given cycle length, as the amount of diastolic filling in AF depends on the random cycle length of the preceding beat. In summary, windowing in AF does not provide a clinically significant difference in $\mathrm{EF}$ determination. If cycle length windowing is used, the exact location of the window is not critical.
\end{abstract}

* Current address: William Beaumont Hospital, Royal Oak, Michigan, USA

Offprint requests to: Jerold $\mathrm{W}$. Wallis at his current address: Mallinckrodt Institute of Radiology, Washington University School of Medicine, 510 South Kingshighway St. Louis, MO 63110, USA
Key words: Atrial fibrillation - Gated blood-pool imaging - Ventriculography - Ejection fraction

Eur J Nucl Med (1991) 18:23-27

\section{Introduction}

In gated blood pool studies, images from corersponding portions of the cardiac cycle are summed to enhance counting statistics, usually using an ECG gate for synchronization. This assumes that individual cardiac cycles are uniform in length and shape, an assumption that is invalid in atrial fibrillation (AF).

Various methods have been proposed to deal with problems of cycle length variation (Bacharach et al. 1979; Bacharach and Green 1982). One method that is gaining increasing acceptance is cycle length windowing, either by utilizing list mode acquisition or dynamic arrhythmia filtration (Wallis et al. 1986). With this technique one can select a narrow range of $R-R$ intervals (or equivalently, a narrow range of heart rates), and only beats within this range or "window" are included in the final study. In people with normal sinus rhythm, dynamic arrhythmia filtration can be employed by observing the patient for 1 or more min and selecting a window centered about the peak of the observed cycle length histogram (Fig. 1).

Dynamic arrhythmia filtration has several potential advantages in atrial fibrillation: [1] Cycles are of uniform length, eliminating the count drop-off in later frames which can be distracting during interpretation and prevents accurate assessment of diastolic filling. [2] The location of end-systole for determination of the ejection fraction (EF) may be more easily assessed, as this can become obscured with severe count drop-off. How- 


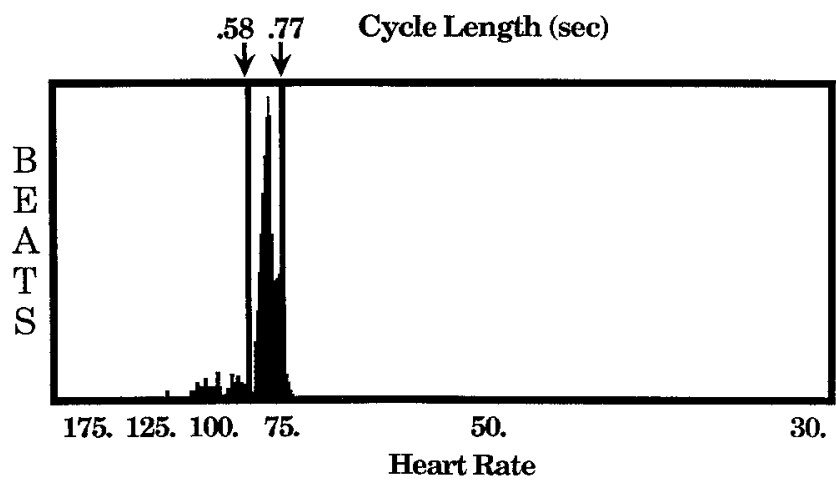

Fig. 1. Cycle length or heart rate histogram in a normal subject, showing the distribution of cycle lengths during an observation period. The operator has chosen a window (upper and lower limits shown at arrows) to determine which cycle lengths will be included in the gated study

ever, the choice of the cycle length window position for patients with AF is less clear. As is seen in Fig. 2, such patients may have a wide histogram without obvious peaks. While one can arbitrarily choose a window position, the question arises as to the effect of this choice. If the EF varies greatly with cycle length, then the choice of window position could substantially affect the value obtained. Furthermore, if the patient is being followed with sequential exams, then an identical window choice would be needed to ensure accurate comparison between studies. Despite the fact that many centres utilize some form of beat filtration in the clinical examination of patients with $\mathrm{AF}$, the use of dynamic arrhythmia filtration to perform cycle length windowing in AF has not been formally examined in the literature. The goals of this study were therefore [1] to assess differences of EF in AF with and without windowing and [2] to determine how $\mathrm{EF}$ varies with cycle length in patients with $\mathrm{AF}$.

\section{Methods}

Twenty patients with $\mathrm{AF}$ at the time of a resting gated blood pool study were prospectively examined. All patients had 12 lead ECGs obtained prior to and ECG monitoring throughout the study. Following intravenous injection of stannous pyrophosphate, patients underwent in vivo labelling with $25 \mathrm{mCi}$ pertechnetate Tc $99 \mathrm{~m}$ (Thrall et al. 1978). Electrocardiogram gated scintigraphic images were obtained from a "best septal" left anterior oblique view with $10-15^{\circ}$ caudal tilt.

The patients were observed for 1-2 min and a cycle length histogram generated. A computer system capable of simultaneous multiple window dynamic arrhythmia filtration acquisition (Medical Data Systems A3) was employed. Five to seven windows were chosen on the visual display to span the majority of the cycle length histogram, utilizing approximately $10 \%$ windows (as exemplified in Fig. 2). Forward gating was employed, cycle selection was based on the length of the current beat, and 16 frames per cardiac cycle were utilized in this study. An acquisition time of 10-15 min was necessary to improve counting statistics, and all windows analysed contained data from at least 100 cardiac cycles.
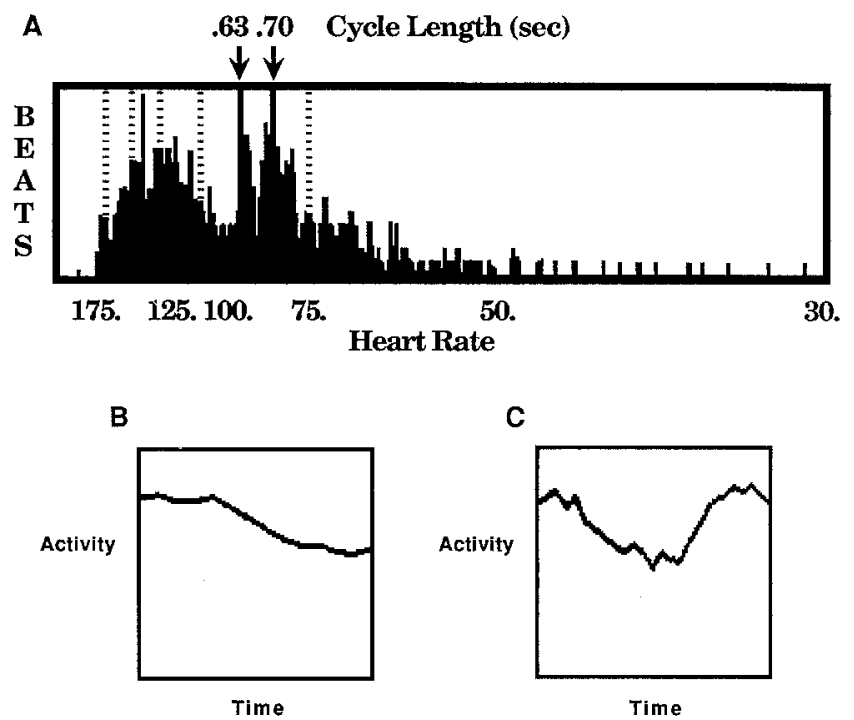

Fig. 2. A Cycle length or heart rate histogram in a patient with atrial fibrillation and a widely varying cycle length. The operator has chosen an arbitrary window (upper and lower limits are the solid bars shown at arrows) to determine which cycle lengths will be included in the gated study. B Time-activity curve using all cycles. C Time-activity curve using data from the selected window. Dotted lines in A show other potential windows; these were collected separately as part of the study acquisition

The EF for each window was determined with semi-automatic commercial software (Medical Data Systems A3) using a combined second derivative and threshold method of edge detection with a variable region of interest. The algorithm automatically identified a background region adjacent and slightly inferior to the left ventricle (LV). Background activity was subtracted from LV activity in each frame to produce the time-activity curve. Cycle length range for each window was recorded for later analysis, along with the number of cycles that fell within that window during acquisition. Count data from the individual cycle length windows were combined to yield data equivalent to a non-windowed study, and the EF determined from this "non-windowed" study as well.

The EF from the non-windowed study in each patient was compared with the individual windowed EF values and with the weighted average of the windowed values for that patient, weighting each EF value by the number of cardiac cycles that fell within that window. This weighted average will hereafter be referred to as the average windowed $\mathrm{EF}$.

\section{Results}

Mean heart rate in the population was 94 (range 58124). Individual windows contained an average of 176 $(\mathrm{SD} \pm 64)$ cycles and mean window with was $8.8 \%$ ( $\mathrm{SD} \pm$ 3.06). No trend of EF was seen with window width or number of cycles per window.

\section{Ejection fraction differences with and without windowing}

A plot of average windowed vs. non-windowed EF for all patients is shown in Fig. 3. The ordinate value of 


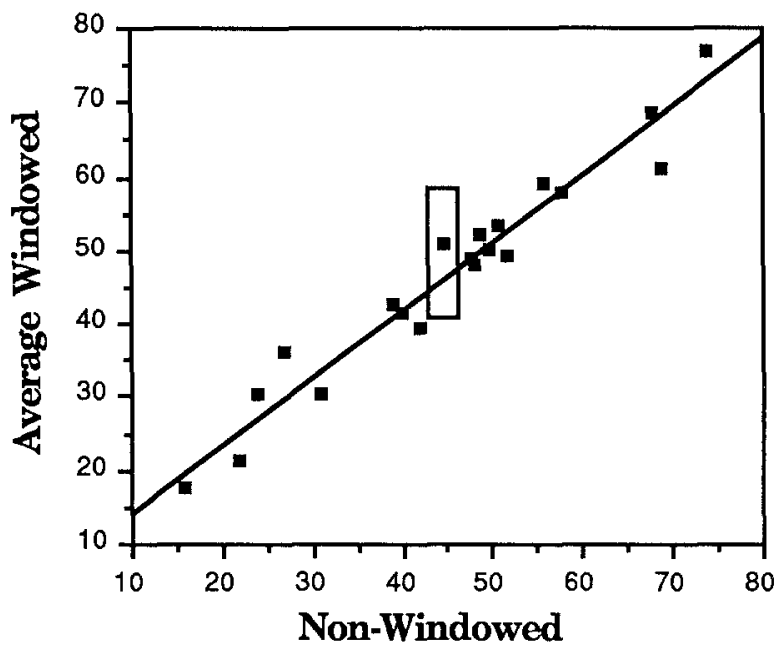

Fig. 3. Average windowed vs. non-windowed ejection fraction $(\mathrm{EF})$ : plot comparing an average of the windowed EF values for each patient weighted by the number of cycles within each window vs. the non-windowed EF for that patient $(r=0.97$, s.e.e. 3.5$)$. Point in the small rectangle represents data from a single patient whose data will be followed through several figures

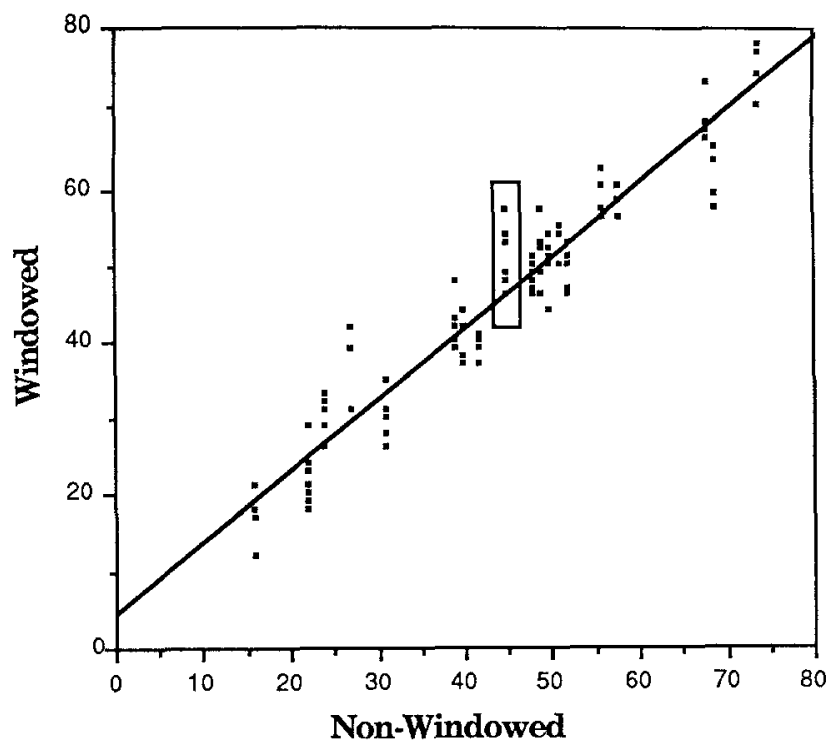

Fig. 4. Individual windowed vs. non-windowed EF: data from a single patient is shown within the box, with the individual window EF values from this patient plotted on the ordinate vs. his nonwindowed values on the abscissa. Data from the other 19 patients are displayed in the remainder of the plot $(r=0.96$, s.e.e. 4.3$)$

each point in this plot represents the weighted average of the windowed EF values in each patient; the individual window values showing the range of $\mathrm{EF}$ values in each patient are depicted in Fig. 4. The windowed EF values were slightly higher than the non-windowed values (mean windowed $\mathrm{EF}$ : 46.6 , mean non-windowed $\mathrm{EF}: 45.5)$, but the difference was not statistically significant $(P=0.16)$. The correlation was quite good between the non-windowed and cycle length windowed techniques $(r=0.97$, s.e.e. 3.5$)$.

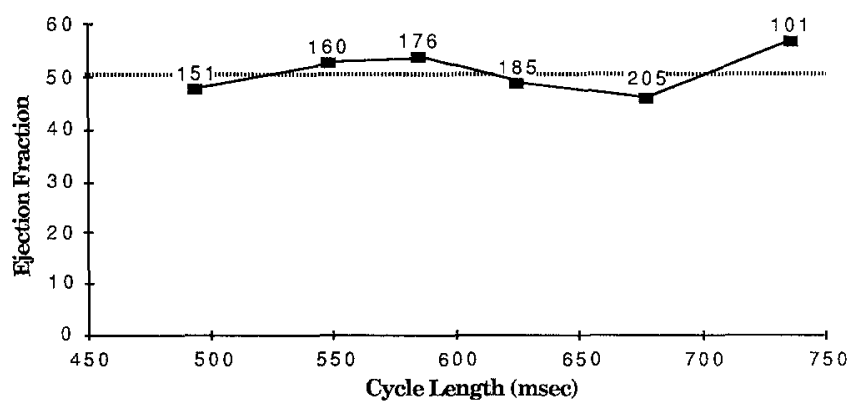

Fig. 5. EF vs. cycle length in a sample patient: EF from the individual windows in a sample patient is plotted vs. the cycle length corresponding to the center of the window. Dotted line represents the average windowed EF in this patient. The numbers above each point indicate the number of cardiac cycles contributing to that particular EF determination

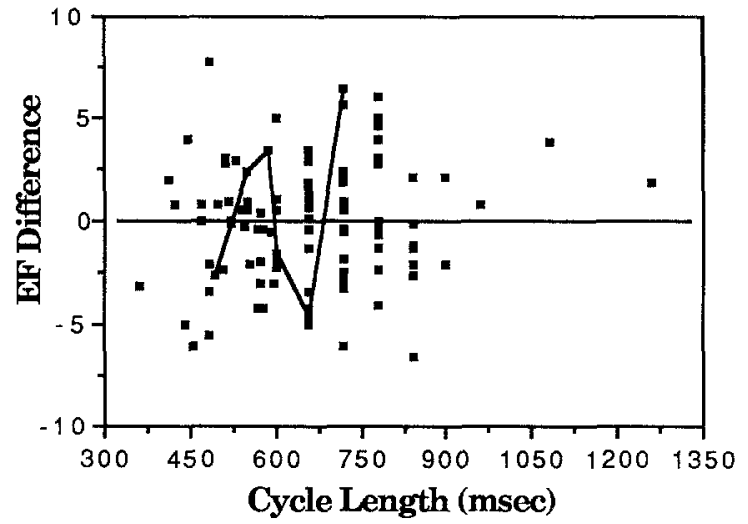

Fig. 6. EF vs. cycle length in all subjects, plotted after aligning the patients by their average windowed EF. For illustrative purposes, the points representing the same patient as in Fig. 5 have been connected, though the scale has been expanded

\section{Ejection fraction vs. cycle length}

A plot of EF vs. cycle length in a single representative patient is shown in Fig. 5. It reveals a small amount of variation of EF with different cycle length windows (SD 4.1 EF units) but without an overall trend of increase or decrease in EF with cycle length. Other patients showed a similar or lesser amount of variation (mean SD 3.2 EF units), but the pattern of variation was not consistent across patients. Combined data for all patients are shown in Fig. 6. No linear or non-linear relationship of EF with cycle length was observed. A plot of EF vs. percentage mean cycle length (not shown) similarly lacked a trend of EF with cycle length.

\section{Discussion}

In a majority of patients with normal sinus ryhthm with a narrow range of $R-R$ intervals, comparison of singlebeat time-activity curves with curves derived from gated images has shown an adequate correspondence for sys- 
tolic parameters (Bacharach et al. 1980). Gated images, however, have been shown occasionally to underestimate LV function parameters, with the largest error in the measurement of diastolic function (Bacharach et al. 1980). These differences can be attributed in part to physiological variation in the shape and length of the LV time-activity curve, with subsequent distortion in the shape of the curve when individual cycles are summed (Juni et al. 1983). Such differences will be greatest when the cycle length varies most widely during data acquisition, as in the case of AF. In patients with varying R-R intervals, frames an equal distance from the ECG gate may represent different portions of the various cardiac cycles. Furthemore, due to variation in cycle length, later frames in the image sequence will have fewer cycles contributing counts to them than do earlier frames, resulting in the decline of counts at the end of the time-activity curve often seen in clinical studies.

Several approaches have been taken toward minimizing the distortion introduced by variation in cardiac cycles, including beat rejection after premature contractions, combined forward/backward gating schemes and beat selection techniques such as list mode acquisition and dynamic arrhythmia filtration (Bacharach et al. 1979; Bacharach and Green 1982; Johnson et al. 1985; Wallis et al. 1986; Juni and Chen 1988). In all the beat selection techniques, only count data from cycles that fall within a preselected cycle length interval (or heart rate range) are added together to form the final gated study.

Dynamic arrhythmia filtration utilizes multiple memory buffers in the computer memory to hold image data from the current cycle until that cardiac cycle is completed. At this point, the cycle length is analyzed, and data from that cycle is added to the final image only if it falls within a preselected $R-R$ range. The system utilized in this project allowed several different gated blood pool studies to be generated simultaneously, each with a different R-R range.

Use of these methods requires selection for a $R-R$ interval to determine which beats are included in the EF analysis. Based on physiological principles and catheterization data, one might expect a substantial variation of EF with cycle length. Beats following a premature ventricular contraction have been shown to have an increased degree of contractility (Ganong 1973), and this post-extra-systolic potentiation has been demonstrated scintigraphically (Kalff et al. 1982). Studies suggest that increasing beat intervals allows more time for both diastolic filling (Starling effect) and recirculation of sequestered calcium ions (Seed et al. 1984; Pidgeon et al. 1980). Right atrial pacing studies have shown a fall in EF as the interval between beats is decreased (Erbel et al. 1984).

As noted above, no significant predictable EF variation with cycle length was found in this study. This suggests that exact window choice is not critical in EF deter- mination in the setting of AF and therefore that identical windows need not be reproduced in serial studies of a given patient. It is, however, necessary to explain this somewhat surprising result in view of the physiological evidence for the cycle length dependence of EF.

Physiological data indicate that preload is a major determinant in the degree of ventricular filling, and increased distension of the ventricle will initiate a more forceful contraction according to the Starling principle (Ganong 1973). This ventricular filling actually occurs during the diastolic filling phase of the preceding cardiac cycle. In the case of normal sinus rhythm, the length of the preceding cycle will be approximately equal to that of the current cycle. Windowing on the current cycle will therefore have the effect of obtaining a uniform preceding beat length, with uniform rate-dependent ventricular filling.

In $\mathrm{AF}$, there is a constantly varying cycle length. Even though one selects beats of uniform length with dynamic arrhythmia filtration, such beats will have a preceding beat of random length and hence a varying amount of diastolic filling. If cycle length is truly random, this will tend to average out the effect of diastolic filling over many beats, producing a mean EF for that cycle length. The same effect takes place for each window, resulting in similar EF values. Had windowing taken place on the length of the preceding beat, a much larger cycle length dependence of EF would likely have been observed. Data from studies of subjects with irregular cycle lengths in which the EF was plotted against the length of the previous beat (van Gelder and Dormehl 1983; Bacharach et al. 1981; Schneider et al. 1983) have confirmed a rate dependency. Many commercial systems do carry out windowing or cycle selection based on the length of the previous beat, and cycle length dependence of the EF would be expected in this setting. Windowing on the prior beat, however, reintroduces the problem of declining curves from frame drop-off due to variation in the current cycle length. If windowing were performed on both the present and previous beats, it is likely that no count drop-off would be present, and cycle length dependence of EF would be seen. However, accepting image data only when two consecutive beats were of a specified length would be impractical, as the acquisition time to obtain a study with adequate counting statistics would be quite lengthy.

The similarity in EF values between the windowed and non-windowed studies suggests that corresponding portions of the cardiac cycles were able to add correctly despite the difference in cardiac cycle length. Review of the data from this study shows that for the individual windows, end-systole occurred either in the same frame as in the non-windowed study or within one frame in over $90 \%$ of cases, supporting this hypothesis. Furthermore, studies have shown that when the cycle length varies, the major change is in the length of the diastolic phase (Juni et al. 1983; Hammermeister and Warbasse 
1974), and that in AF, $98 \%$ of cycles are intact through the time of peak filling (Bacharach et al. 1981). The fact that cycles are nearly always intact past the point of end-systole and that there is little variation in time to end-systole permits the first portion of the cardiac cycle to add together properly during gated imaging, in spite of $\mathrm{R}-\mathrm{R}$ variation. These factors also make forward gating more appropriate than backward gating in the evaluation of systolic function.

Variation exists between windows in a given patient as noted above, although this variation was not predicted by cycle length or window width. Such variation is in part due to counting statistics, as even with prolonged acquisition a limited number of cycles are contained within any given window. In view of the above findings, it may not be beneficial to use an extremely narrow window for EF determination in patients with $\mathrm{AF}$.

One limitation of this study is the lack of a "true" $\mathrm{EF}$ value. Ideally in this type of analysis, one would have available a gold standard EF against which to measure the accuracy of the calculated EF. Unfortunately, such is not readily available due to the beat-to-beat $\mathrm{EF}$ variation that exists in AF. Geometrically based catheterization techniques or count-based first-pass nuclear studies would measure the EF of only a few beats and may not yield a representative picture of cardiac function. A better measure of cardiac function would be individual beat EF, averaged over all beats. In the limited data in the literature that did utilize individual beat EF values using a cardiac probe, gated values in $\mathrm{AF}$ were found to be similar to an average of the individual beat EF values (Bacharach et al. 1981; Schneider et al. 1983). Windowing should minimize distortion due to varying $R-R$ intervals, while still effectively producing an average $\mathrm{EF}$ for cycles included in that window. An average of the $\mathrm{EF}$ values computed for each window weighted by the number of cycles that fell in that window, as used in this study, should give an average EF for all cardiac cycles during the acquisition period. While the beat-to-beat EF may vary substantially due to variable diastolic filling, the average EF still provides an overall measure of cardiac function in patients with AF.

These results apply only to windowing on the current beat length, as is done in dynamic arrhythmia filtration. As noted above, techniques that window on the previous beat length, including post-beat rejection, might be expected to show a trend in EF based on the selected cycle length window.

In conclusion, cycle length windowing in AF using dynamic arrhythmia filtration does not provide a clinically significant difference in EF determination compared with non-windowed acquisition. If cycle length windowing is used, the exact location of the window ist not critical.

\section{References}

Bacharach SL, Green MV (1982) Data processing in nuclear cardiology: measurement of ventricular function. IEEE Trans Nucl Sci NS-29 (4): 1343-1353

Bacharach SL, Green MV, Borer JS (1979) Instrumentation and data processing in cardiovascular nuclear medicine: evaluation of ventricular function. Sem Nucl Med 9:257-274

Bacharach SL, Green MV, Borer JS, Ostrow HG, Bonow RO, Farkas SP, Johnston GS (1980) Beat-by-beat validation of ECG gating. J Nucl Med 21:307-313

Bacharach SL, Green MV, Bonow RO, Findley SL, Ostrow HG, Johnston GS (1981) Measurement of ventricular function by ECG gating during atrial fibrillation. J Nucl Med 22:226-231

Erbel R, Schweizer P, Krebs W, Langen H-J, Meyer J, Effert S (1984) Effects of heart rate changes on left ventricular volume and ejection fraction: a 2-dimensional echocardiographic study. Am J Cardiol 53:590-597

Ganong WF (1973) Review of medical physiology. Lange Medical, Los Altos, CA, USA

Hammermeister KE, Warbasse JR (1974) The rate of change of left ventricular volume in man: diastolic events in health and disease. Circulation 49:739-747

Johnson TK, Boudreau RJ, Loken MK (1985) Dependence of measured diastolic function on acquisition mode: gated vs. list. J Nucl Med 26:107

Juni JE, Chen C (1988) Effects of gating modes on the analysis of left ventricular function in the presence of heart rate variation. J Nucl Med 29:1272-1278

Juni JE, Froelich JE, McMeekin J, Bourdillon P, Rocchini A, Botti J, Buda A, Pitt B (1983) The effects of heart rate variability on scintigraphic measurements of diastolic function. Circulation $68:$ III-246

Kalff V, Chan W, Rabinovitch M, O'Neill W, Stewart J, Walton J, Thrall JH, Pitt B (1982) Radionuclide evaluation of postextrasystolic potentiation of left ventricular function induced by atrial and ventricular stimulation. Am J Cardiol 50:106-111

Pidgeon J, Lab M, Seed A, Elzinga G, Papadoyannis D, Noble MIM (1980) The contractile state of cat and dog heart in relation to the interval between beats. Circ Res 47:559-567

Schneider J, Berger HJ, Sands MJ, Lachman AB, Zaret BL (1983) Beat-to-beat left ventricular performance in atrial fibrillation: radionuclide assessment with the computerized nuclear probe. Am J Cardiol 51:1189-1195

Seed WA, Noble MI, Walker JM, Miller GAH, Pidgeon J, Redwood D, Wanless R, Franz MR, Schoettler M, Schaefer J (1984) Relationship between beat-to-beat interval and the strength of contraction in the healthy and diseased human heart. Circulation 70(5): 799-805

Thrall JH, Freitas JE, Swanson E, Rogers WL, Clare JM, Brown ML, Pitt B (1978) Clinical comparison of cardiac blood pool visualization with technetium $99 \mathrm{~m}$ red blood cells labeled in vivo and with technetium $99 \mathrm{~m}$ human serum albumin. J Nucl Med 19:796

van Gelder AL, Dormehl IC (1983) The influence of cycle length on the radionuclide left ventricular volume curve acquired with a scintillation camera and ECG triggered data processor. Nuklearmedizin 22(3): 145-148

Wallis JW, Wu-Connolly L, Rocchini AP, June JE (1986) Dynamic arrhythmia filtration for gated blood pool imaging: validation against list mode technique. $\mathrm{J}$ Nucl Med 27:1347-1352 